\title{
Tenofovir and remdesivir ensemble docking with the SARS-CoV-2 polymerase and template-nascent RNA
}

\author{
Pablo M De Salazar ${ }^{1}$, Javier Ramos ${ }^{2}$, Victor L Cruz ${ }^{2}$, Rosa Polo ${ }^{3}$, Julia Del Amo ${ }^{3}$, and \\ Javier Martínez-Salazar ${ }^{2}$ \\ ${ }^{1}$ Center for Communicable Disease Dynamics, Department of Epidemiology, Harvard \\ School of Public Health, Boston, US. \\ ${ }^{2}$ Byophym group, Institute for the Structure of Matter, Spanish National Research Council \\ (CSIC), Madrid, Spain \\ ${ }^{3}$ National Plan Against AIDS, Ministry of Health, Madrid, Spain
}

September 28, 2020

\begin{abstract}
Repositioning of remdesivir and tenofovir against COVID-19 has shown only partial evidence of improving clinical outcomes, in clinical trials and observational studies respectively. The rationale behind this inconsistent efficacy remains unknown. Here, we developed an ensemble docking approach for the active triphosphate forms of both antivirals with the SARS-CoV-2 polymerase and the RNA chain complex, under the hypothesis that clinical observation could rely on the specificities of the drug-target interaction. Our model framework allowed accurate reconstruction of the remdesivir ensemble, which presented the strongest binding affinity and pose stability close to the natural counterpart dATP. We further observed a set of features of the tenofovir complex that suggests functional yet suboptimal interaction, likely resulting in limited viral inhibition in the absence of high intracellular concentration at target tissues. Our findings provide rationale for the mixed effectiveness of tenofovirbased compounds against SARS-CoV-2 and underscore the relevance of the intracellular availability of the nucleotide analogues relative to viral tropism.
\end{abstract}

\section{Introduction}

Definitive therapies for preventing and treating COVID-19, the disease caused by SARS-CoV-2, are still lacking [1]. Worldwide deployment of potential effective vaccines will likely take several months if not years [2]. Meanwhile, there is an urgent need for alternatives that help reduce mortality and protect the most vulnerable. Repositioning (finding a new therapeutic use for an already known drug) is the fastest option to deliver treatment because safety and tolerance have already been ascertained; however, deciding which drugs should be prioritized is challenging, as is determining the best pre-clinical approaches to develop before clinical evaluation is reached [3].

To date, only two repositioned antivirals have shown partial evidence in preventing COVID-19 morbidity: remdesivir as treatment for severe COVID-19 patients in a randomized clinical trial [5] and tenofovir (as its prodrug tenofovir disoproxil fumarate, TDF) in a retrospective observational study following HIV individuals under antivirals [4]. Remdesivir and tenofovir were originally designed to inhibit the ATP polymerization into the growing nucleic acids chain in Ebola virus polymerase [6] and HIV reverse transcriptase [7], respectively.

A recent randomized clinical trial in the US reported shorter recovery for COVID-19 hospitalized individuals treated with remdesivir compared to placebo, but the study did not show a significant decrease in mortality [5]. However, an earlier trial in China reported no differences in clinical outcomes for treatment with 
remdesivir versus placebo, although this trial had inadequate statistical power due to not reaching planned sample size [8]. On the other hand, evidence of the pre-exposure prophylactic efficacy of the nucleotide analogous tenofovir, in particular of TDF in combination with emcitribacine (FTC), has been suggested in a recent observational study evaluating SARS-CoV-2 infection outcomes among HIV patients under antiretroviral therapy in Spain [4]. Individuals taking TDF/FTC for their HIV infection showed significantly lower risk of SARS-CoV-2 infection and hospitalization compared with individuals taking other antivirals or taking the tenofovir-based prodrug tenofovir alafenamide fumarate (TAF) with FTC. A similar protective trend for TDF/FTC on COVID-19 mortality was observed in South Africa when compared to zidovudine or abacavir-based regimes [9]. Nevertheless, the findings still need to be confirmed by randomized clinical trials [10]. This discrepancy between the different tenofovir prodrugs leading to mixed effectiveness is unexplained, beyond the potential existence of confounding factors in the analysis, which has been partly excluded for the Spanish cohort [11].

In order to achieve viral inhibition, nucleic acid analogous must bind the viral polymerase inside infected cells after metabolizing into their active forms. In particular, for tenofovir and remdesivir to block SARS-CoV-2 replication it is required that their active triphosphate forms are capable of strongly binding to the viral RNA polymerase in the presence of nascent RNA, substituting their natural counterpart deoxyadenosine triphosphate dATP, which in turn leads to incorporating the antimetabolite in the chain and terminating polymerization $[12,13]$. Thus, affinity and stability of the antivirals in the active pocket of the viral polymerase define any further inhibition.

Here, we designed an ensemble docking approach derived from crystal structures and implemented with extensive sampling using volunteer distributed computation, to predict the binding location, affinity and stability of the active forms remdesivir-triphosphate, tenofovir-diphosphate, and dATP with the SARSCoV-2 RNA-dependent RNA polymerase (RdRp-CoV2) and the template-nascent RNA chain complex, and to determine potential limitations arising from their molecular structures.

\section{Results}

We performed ensemble docking simulations using the cryo-electron microscopy structure of the nsp12 in complex with template-nascent RNA and each of the following: remdesivir-triphosphate, tenofovir-diphosphate and dATP.

Computed relative binding energies $\left(\Delta \mathrm{G}_{\text {binding }}\right)$ showed that remdesivir presented the strongest binding (precision range -8.7 to $-8.5 \mathrm{kcal} / \mathrm{mol}$ ), followed by dATP $(-8.6$ to $-8.4 \mathrm{kcal} / \mathrm{mol}$ ) and tenofovir $(-7.7$ to -7.5 $\mathrm{kcal} / \mathrm{mol}$ ). The three ligands shared the same binding site and were forced to adopt a different conformation in order to allow the insertion of the incoming nucleotide into the nascent RNA. Docking structures of dATP, remdesivir, and tenofovir ensembles are displayed in Figure 1. Including the RNA chains/magnesium ions in the analysis reduced the computed $\Delta \mathrm{G}_{\text {binding }}$ for all three ligands while maintaining a similar affinity gradient (see supplementary material, S2). We further observed that the main binding sites of the ligands were situated in a different pocket than were the binding sites of the ensemble without template-nascent RNA (RdRp-CoV2-NoRNA).

We observed that the lowest binding energy conformations did not always show the expected interactions between the template-nascent RNA strands and the analyzed ligand. Thus, we searched for the poses with

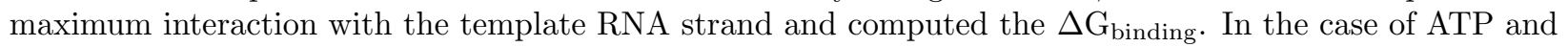
remdesivir, we found that those poses remain close to the lowest computed $\Delta \mathrm{G}_{\text {binding }}$ (ca. $0.1 \mathrm{kcal} / \mathrm{mol}$ ), while the tenofovir ensemble showed a greater difference between the pose with RNA interactions and the lowest $\Delta \mathrm{G}_{\text {binding }}$ poses (ca. $0.5 \mathrm{kcal} / \mathrm{mol}$ ). The most probable driver for the lower $\Delta \mathrm{G}_{\text {binding }}$ of remdesivir compared to ATP is the extra H-bond between the cyano group and the U927 nucleotide of the nascent RNA strand.

For the three ligands, both the Mg2+ ion and the charged ARG621 helped to stabilize the position of the triphosphate moiety by electrostatic and salt-bridge interactions. Further, typical H-bonds between the uracil nucleotide of the template RNA (U930) strand and (like) adenosine rings presented in the three 
ligands were observed supporting the formation of binding complex between the incoming ligand and the template RNA strand, previous to the insertion into the nascent RNA strand. Additionally, hydrophobic interactions between the U927 and A931 in the template and nascent RNA strands helped to better stabilize the dATP and remdesivir ensembles. However, for tenofovir the observed interactions with the nucleotides in the nascent RNA were different. In particular, an H-bond was formed between the adenine ring of tenofovir and U927 nucleotide. This arrangement hindered the formation of the hydrophobic interactions observed in the dATP and remdesivir ensembles. Interestingly, a lowest binding energy pose far from the center site of inhibition was found for tenofovir, but not for remdesivir nor dATP (see Figure S2), which suggests a non-functional competitive site for tenofovir.

In summary, ensemble molecular docking was performed using dATP, remdesivir-triphosphate, and tenofovirdiphosphate binding the RdRp-CoV2 target receptor with template-nascent RNA. The remdesivir ensemble was accurately reconstructed, compared to previous models, and the antiviral active form presented the strongest binding affinity closely followed by dATP. Further, tenofovir interacted in the same pocket as remdesivir/dATP but presented significantly weaker binding affinity and pose stability, and a relatively stable non-functional binding with the RdRp-CoV2 close to the active pocket.

\section{Discussion}

First, our findings show that similar intracellular concentrations of remdesivir relative to dATP can effectively inhibit SARS-CoV2 replication. Indeed, Gordon et al. reported high selectivity of the antiviral over incorporation of dATP using purified active RdRp-CoV2 [12]. Further, remdesivir has been reported to inhibit SARS-CoV-2 in lung cells at submicromolar concentrations in vitro [22]. Moreover, similar binding site for remdesivir in the SARS-CoV-2 polymerase have been reported recently by two different approaches, based on a 3D-structural alignment using other virus polymerases [23] and a constrained minimization and conformational search on the RdRp-CoV2 active site [22,23]. In fact, our model provided the specific molecular interactions supporting the binding site (see supplementary material S3 for detailed description). The cyano group in remdesivir, a group not present in the other ligands, allows additional H-bond interactions

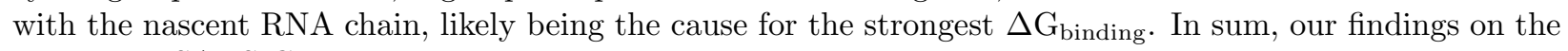
remdesivir-SARS-CoV2 interaction are robustly aligned with recent studies and validates our approach as a reliable framework to evaluate nucleic acids analogues against SARS-CoV2. We conclude that the partial clinical efficacy observed for remdesivir is not the result of molecular interactions with the RdRp-CoV2, given its strong binding affinity and drug target stability. Alternatively, remdesivir effectiveness might rely on unstudied pharmacokinetic properties and/or time to treatment relative to viral replication.

Regarding tenofovir, our approach is the first to include template-nascent RNA in the ensemble and further improves previous work [24] by using cryo-EM structures instead of homology and by more extensive sampling of the ligand poses using volunteer distributed computations. Consistent with our findings, tenofovirdiphosphate has been shown to permanently terminate polymerase extension of nascent RNA when using recombinant RdRp-CoV2 [25]. However, infusion of tenofovir in Vero cell cultures did not inhibit replication of SARS-CoV-2 [26,27], while the use of 3-90 $\mu \mathrm{M}$ of its prodrug TDF yielded a 15-fold reduction of viral genome release [26] . Further, the use of TDF/FTC for treating SARS-CoV-2 infected ferrets led to better clinical scores and lower virus titers in nasal washes compared to a placebo [28]. Worth noting, the prodrug TDF, formulated to increase tenofovir limited bioavailability [29], is known to diffuse passively across cellular membranes [30,31] and further activate intracellularly, as opposed to tenofovir which requires active transportation for intake before activation $[32,33]$. Indeed, higher levels of active metabolite after exposure to TDF versus tenofovir has been consistently reported in several cell types [34-36]. In contrast, the prodrug TAF was formulated to reduce drug-adverse events observed for TDF (which distributes body-wide) by being highly HIV-target-cell specific. TAF is well known to selectively activate and present preferential distribution in lymphatic tissues [37].

Our findings support a suboptimal tenofovir-RdRp-CoV2 interaction compared to remdesivir, predicting that the ensemble is more sensitive to the triphosphate form intracellular concentration. Thus, matching sufficient intracellular availability of tenofovir-diphostate with SARS-CoV2 tropism $[37,38]$ (such as in the 
respiratory tract [39]) is essential in drug-driven containment of viral replication, mirroring HIV prophylaxis [35]. Of note, viral replication can occur in different tissues at different clinical stages [40]. Thus, tenofovir efficacy might highly depend on when it is given relative to the within-host SARS-CoV-2 distribution. Together with the available pharmacokinetic/pharmacodynamic evidence, our findings support that TDF, among tenofovir-based compounds, maximizes efficacy at safe clinical dosage especially when taken before or close after exposure, because of high cellular permeation, effective metabolite activation, and lowselective distribution at viral targeted tissues, laying out a plausible molecular interpretation of the apparent inconsistency of tenofovir-based compounds against SARS-CoV-2 [11]. Finally, our findings support further evaluation of remdesivir as treatment against COVID-19 but also for the prodrug TDF [10] (available as a generic worldwide), in particular as prophylaxis, while underscoring the need for understanding the intracellular availability of the drugs in SARS-CoV2 targeted tissues.

\section{Methods}

\section{Receptor structure}

The three-dimensional structure of the RdRp-CoV2 solved with RNA nascent and template chains as well as with two magnesium ions on the binding site was obtained from the experimental structure deposited with the PDB (Protein Database Bank) entry 7BV1[14]. Nsp7 and nsp8 cofactors were removed from the model. Herein, the receptor is labelled as RdRp-CoV2-RNA.

An ensemble docking approach is considered in this work. This technique has proved to be more efficient than a single-docking experiment on the crystal structure[15,16] . Consequently, the initial receptor structures were subjected to multi-second molecular dynamics simulations, in order to approximate the conformational flexibility on the receptor [15]. Molecular dynamics (MD) details are given as supplementary material, Section S1.

\section{Ligand structures}

The three-dimensional structures of the dATP, and the biologically active forms of the drugs remdesivir triphosphate [6,17] and tenofovir diphosphate[17], were extracted from experimental structures containing these ligands in the PDB. Prior to the docking simulations, these structures were prepared and subjected to geometry optimization using the Becke-Lee-Yang-Parr exchange correlation functional and the triple numerical plus polarization basis set as implemented in the Dmol3 module of Materials Studio (Dassault Systemes BIOVIA, v20.1.0). In all cases, the charge of the molecules was -4, corresponding to the fully ionized triphosphate groups.

\section{Molecular docking}

The receptor and ligand structures were prepared using AutoDockTools v1.5.7 [18] by removing lone atoms, typing atoms to AutoDock atom types (AD4), adding polar hydrogens, removing non-polar hydrogens, and adding Gasteiger charges. The receptor and ligands were treated as rigid and flexible, respectively. The rotatable bonds of the ligands were defined using the Torsion Tree Tools in AutoDockTools. Ligand structures, along with the type of atoms and the rotatable bonds considered in the docking calculations, are shown in the supplementary material (Figure S1). The docking experiments were performed with AutoDock Vina 1.1.2 [18], which can compute binding energies with a precision interval of $+/-0.1 \mathrm{kcal} / \mathrm{mol}$.

To run, AudoDock Vina requires specifying certain parameters, such as the exhaustiveness (the number of runs), energy range, and the number of binding modes. After performing preliminary test calculations to establish the most suitable values, we selected the following parameter values: $20 \mathrm{runs}, 5 \mathrm{kcal} / \mathrm{mol}$ for the energy range, and 20 binding modes. Structures used in the docking are available from the authors upon request.

Extensive searching in the space of protein-ligand docking conformations was supported by the citizen volunteer computing project COVID-PHYM [19] implemented using the BOINC (Berkeley Open Infrastructure for Network Computing) platform [20]. Around 3.2 million poses were collected for each protein (snapshot)- 
ligand pair. Details concerning the analysis of the docked positions are given as supplementary material, Section S2.

For comparison with previous approaches, we also performed ensemble docking using the RdRp-CoV2 solved without RNA using the experimental structure deposited in the PDB entry 7BTF [21](supplementary material, Section S3).

Data availability: All ligand-receptors structures are available from the authors upon request.

\section{References}

1. Kim AY, Gandhi RT. Coronavirus disease 2019 (COVID-19): Management in hospitalized adults. [cited 4 Sep 2020]. Available: https://www.uptodate.com/contents/coronavirus-disease-2019-covid-19-managementin-hospitalized-adults

2. Lurie N, Saville M, Hatchett R, Halton J. Developing Covid-19 Vaccines at Pandemic Speed. N Engl J Med. 2020;382: 1969-1973.

3. Lima WG, Brito JCM, Overhage J, Nizer WS da C. The potential of drug repositioning as a shortterm strategy for the control and treatment of COVID-19 (SARS-CoV-2): a systematic review. Arch Virol. 2020;165: 1729-1737.

4. Del Amo J, Polo R, Moreno S, Díaz A, Martínez E, Arribas JR, et al. Incidence and Severity of COVID19 in HIV-Positive Persons Receiving Antiretroviral Therapy: A Cohort Study. Ann Intern Med. 2020. doi:10.7326/M20-3689

5. Beigel JH, Tomashek KM, Dodd LE. Remdesivir for the Treatment of Covid-19 - Preliminary Report. Reply. The New England journal of medicine. 2020. p. 994.

6. Tchesnokov EP, Feng JY, Porter DP, Götte M. Mechanism of Inhibition of Ebola Virus RNA-Dependent RNA Polymerase by Remdesivir. Viruses. 2019;11. doi:10.3390/v11040326

7. Wassner C, Bradley N, Lee Y. A Review and Clinical Understanding of Tenofovir: Tenofovir Disoproxil Fumarate versus Tenofovir Alafenamide. J Int Assoc Provid AIDS Care. 2020;19: 2325958220919231.

8. Wang Y, Zhang D, Du G, Du R, Zhao J, Jin Y, et al. Remdesivir in adults with severe COVID-19: a randomised, double-blind, placebo-controlled, multicentre trial. Lancet. 2020;395: 1569-1578.

9. Davies M-A. HIV and risk of COVID-19 death: a population cohort study from the Western Cape Province, South Africa. doi:10.1101/2020.07.02.20145185

10. Randomized Clinical Trial for the Prevention of SARS-CoV-2 Infection (COVID-19) in Healthcare Personnel - Full Text View - ClinicalTrials.gov. [cited 4 Sep 2020]. Available: https://clinicaltrials.gov/ct2/show/NCT04334928

11. Del Amo J, Polo R, Moreno S, Díaz A, Martínez E, Arribas JR, et al. Antiretrovirals and risk of COVID-19 diagnosis and hospitalization in HIV-positive persons. Epidemiology. 2020. doi:10.1097/EDE.0000000000001235

12. Gordon CJ, Tchesnokov EP, Woolner E, Perry JK, Feng JY, Porter DP, et al. Remdesivir is a direct-acting antiviral that inhibits RNA-dependent RNA polymerase from severe acute respiratory syndrome coronavirus 2 with high potency. J Biol Chem. 2020; jbc.RA120.013679.

13. Iyidogan P, Anderson KS. Understanding the molecular mechanism of sequence dependent tenofovir removal by HIV-1 reverse transcriptase: differences in primer binding site versus polypurine tract. Antiviral Res. 2012;95: 93-103.

14. Yin W, Mao C, Luan X, Shen D-D, Shen Q, Su H, et al. Structural basis for inhibition of the RNAdependent RNA polymerase from SARS-CoV-2 by remdesivir. Science. 2020;368: 1499-1504. 
15. Amaro RE, Baudry J, Chodera J, Demir Ö, Andrew McCammon J, Miao Y, et al. Ensemble Docking in Drug Discovery. Biophysical Journal. 2018. pp. 2271-2278. doi:10.1016/j.bpj.2018.02.038

16. Evangelista Falcon W, Ellingson SR, Smith JC, Baudry J. Ensemble Docking in Drug Discovery: How Many Protein Configurations from Molecular Dynamics Simulations are Needed To Reproduce Known Ligand Binding? J Phys Chem B. 2019;123: 5189-5195.

17. Lou L. Advances in Nucleotide Antiviral Development from Scientific Discovery to Clinical Applications: Tenofovir Disoproxil Fumarate for Hepatitis B. J Clin Transl Hepatol. 2013;1: 33-38.

18. Morris GM, Huey R, Lindstrom W, Sanner MF, Belew RK, Goodsell DS, et al. AutoDock4 and AutoDockTools4: Automated docking with selective receptor flexibility. J Comput Chem. 2009;30: 2785-2791.

19. Proyecto COVID-PHYM - Fundación Ibercivis. [cited 8 Sep 2020]. Available: https://ibercivis.es/project/proyecto-covid-phym/

20. Anderson DP. BOINC: A System for Public-Resource Computing and Storage. Fifth IEEE/ACM International Workshop on Grid Computing. doi:10.1109/grid.2004.14

21. Gao Y, Yan L, Huang Y, Liu F, Zhao Y, Cao L, et al. Structure of the RNA-dependent RNA polymerase from COVID-19 virus. Science. 2020;368: 779-782.

22. Pruijssers AJ, George AS, Schäfer A, Leist SR, Gralinksi LE, Dinnon KH 3rd, et al. Remdesivir Inhibits SARS-CoV-2 in Human Lung Cells and Chimeric SARS-CoV Expressing the SARS-CoV-2 RNA Polymerase in Mice. Cell Rep. 2020;32: 107940.

23. Shannon A, Le NT-T, Selisko B, Eydoux C, Alvarez K, Guillemot J-C, et al. Remdesivir and SARS-CoV2: Structural requirements at both nsp12 RdRp and nsp14 Exonuclease active-sites. Antiviral Res. 2020;178: 104793.

24. Elfiky AA. Ribavirin, Remdesivir, Sofosbuvir, Galidesivir, and Tenofovir against SARS-CoV-2 RNA dependent RNA polymerase (RdRp): A molecular docking study. Life Sci. 2020;253: 117592.

25. Chien M, Anderson TK, Jockusch S, Tao C, Li X, Kumar S, et al. Nucleotide Analogues as Inhibitors of SARS-CoV-2 Polymerase, a Key Drug Target for COVID-19. J Proteome Res. 2020. doi:10.1021/acs.jproteome.0c00392

26. Liu S, Lien CZ, Selvaraj P, Wang TT. Evaluation of 19 antiviral drugs against SARS-CoV-2 Infection. doi:10.1101/2020.04.29.067983

27. Clososki G, Soldi R, da Silva R, Guaratini T, Lopes J, Pereira P, et al. Tenofovir Disoproxil Fumarate: New Chemical Developments and Encouraging in vitro Biological Results for SARS-CoV-2. Journal of the Brazilian Chemical Society. 2020. doi:10.21577/0103-5053.20200106

28. Park S-J, Yu K-M, Kim Y-I, Kim S-M, Kim E-H, Kim S-G, et al. Antiviral Efficacies of FDA-Approved Drugs against SARS-CoV-2 Infection in Ferrets. MBio. 2020;11. doi:10.1128/mBio.01114-20

29. Robbins BL, Srinivas RV, Kim C, Bischofberger N, Fridland A. Anti-human immunodeficiency virus activity and cellular metabolism of a potential prodrug of the acyclic nucleoside phosphonate 9-R(2-phosphonomethoxypropyl)adenine (PMPA), Bis(isopropyloxymethylcarbonyl)PMPA. Antimicrob Agents Chemother. 1998;42: 612-617.

30. Huttunen KM, Raunio H, Rautio J. Prodrugs-from serendipity to rational design. Pharmacol Rev. 2011;63: 750-771.

31. Arimilli M, Kim C, Dougherty J, Mulato A, Oliyai R, Shaw J, et al. Synthesis, in Vitro Biological Evaluation and Oral Bioavailability of 9-[2-(Phosphonomethoxy)Propyl]Adenine (PMPA) Prodrugs. Antiviral Chemistry and Chemotherapy. 1997. pp. 557-564. doi:10.1177/095632029700800610 
32. Kohler JJ, Hosseini SH, Green E, Abuin A, Ludaway T, Russ R, et al. Tenofovir renal proximal tubular toxicity is regulated by OAT1 and MRP4 transporters. Lab Invest. 2011;91: 852-858.

33. Uwai Y, Ida H, Tsuji Y, Katsura T, Inui K-I. Renal transport of adefovir, cidofovir, and tenofovir by SLC22A family members (hOAT1, hOAT3, and hOCT2). Pharm Res. 2007;24: 811-815.

34. Taneva E, Crooker K, Park SH, Su JT, Ott A, Cheshenko N, et al. Differential Mechanisms of Tenofovir and Tenofovir Disoproxil Fumarate Cellular Transport and Implications for Topical Preexposure Prophylaxis. Antimicrob Agents Chemother. 2015;60: 1667-1675.

35. Patterson KB, Prince HA, Kraft E, Jenkins AJ, Shaheen NJ, Rooney JF, et al. Penetration of tenofovir and emtricitabine in mucosal tissues: implications for prevention of HIV-1 transmission. Sci Transl Med. 2011;3: 112re4.

36. Van Rompay KKA, Babusis D, Abbott Z, Geng Y, Jayashankar K, Johnson JA, et al. Compared to subcutaneous tenofovir, oral tenofovir disoproxyl fumarate administration preferentially concentrates the drug into gut-associated lymphoid cells in simian immunodeficiency virus-infected macaques. Antimicrob Agents Chemother. 2012;56: 4980-4984.

37. Lee WA, He G-X, Eisenberg E, Cihlar T, Swaminathan S, Mulato A, et al. Selective intracellular activation of a novel prodrug of the human immunodeficiency virus reverse transcriptase inhibitor tenofovir leads to preferential distribution and accumulation in lymphatic tissue. Antimicrob Agents Chemother. 2005;49: $1898-1906$.

38. Chu H, Chan JF-W, Yuen TT-T, Shuai H, Yuan S, Wang Y, et al. Comparative tropism, replication kinetics, and cell damage profiling of SARS-CoV-2 and SARS-CoV with implications for clinical manifestations, transmissibility, and laboratory studies of COVID-19: an observational study. Lancet Microbe. 2020;1: $\mathrm{e} 14-\mathrm{e} 23$.

39. Twigg HL, Schnizlein-Bick CT, Weiden M, Valentine F, Wheat J, Day RB, et al. Measurement of antiretroviral drugs in the lungs of HIV-infected patients. HIV Ther. 2010;4: 247-251.

40. Gallagher ME, Brooke CB, Ke R, Koelle K. Causes and Consequences of Spatial Within-Host Viral Spread. Viruses. 2018;10. doi:10.3390/v10110627

41. Salentin S, Schreiber S, Joachim Haupt V, Adasme MF, Schroeder M. PLIP: fully automated proteinligand interaction profiler. Nucleic Acids Research. 2015. pp. W443-W447. doi:10.1093/nar/gkv315

Acknowledgements: We thank Francisco Sanz García and Daniel Lisbona of the Ibercivis Foundation for their assistance provided in the use of the BOINC platform. We also thank Miguel Hernán and Jose Ramón Arribas for their technical advice. Finally, we acknowledge the volunteers who contributed to the COVID-PHYM project using BOINC distributed computing networks.

Competing interests: Authors declare no competing interests.

Author's contribution: PMD, JR, JMS, JDA and RP conceived the idea. PMD, JR, and JMS designed the ensemble docking model. JR and VLC performed the computations. PMD, JR, JMS developed the analysis and wrote the manuscript with support of VCL, RP and JDA.

Funding: J. R. was supported by the CSIC (PIE202050E017) for financial support. PMD was supported by the Ramon Areces Foundation, postdoctoral grant.

Material and correspondence: material request and correpondence should be adressed to PMD (pablom@hsph.harvard.edu) and JR (j.ramos@csic.es).

Figure 1 Analysis of the interactions between the ATP, remdesivir, and tenofovir ligands with the RdRp receptor containing template-nascent RNA and $\mathrm{Mg}$ ions. 

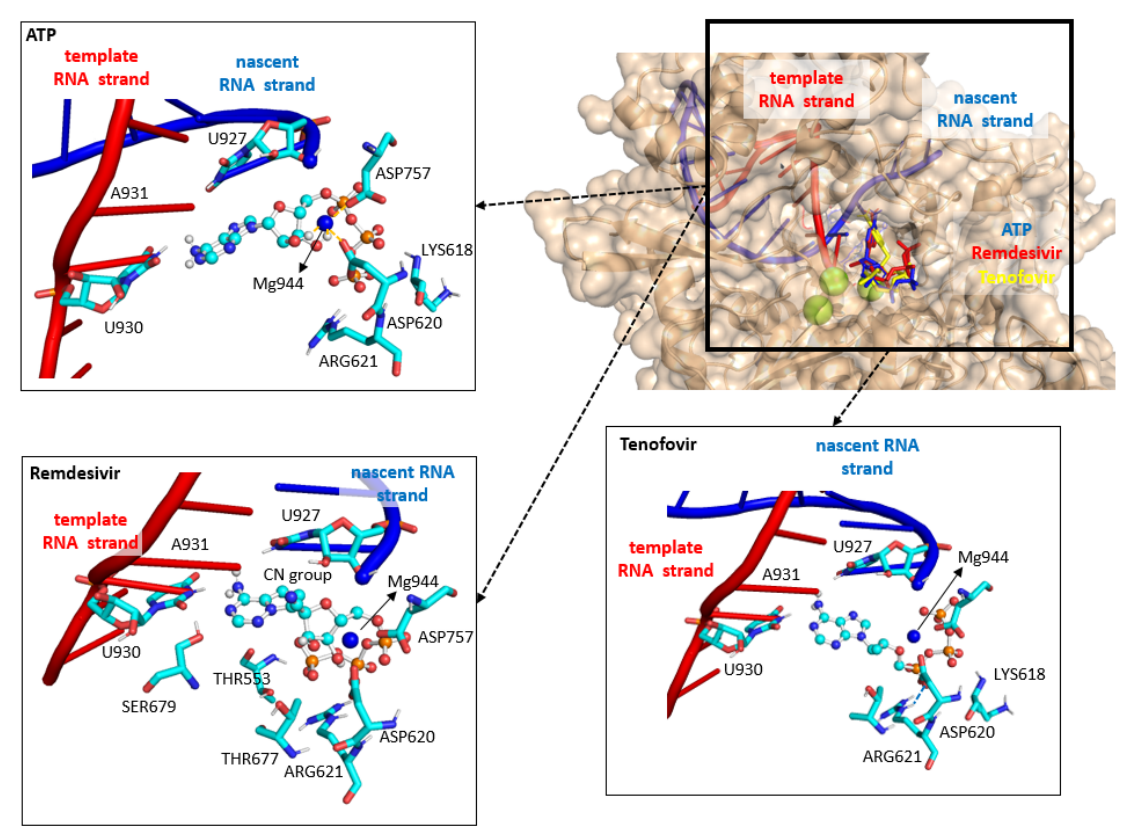

Figure 1: Ligands are displayed as ball-and-stick; RNA and amino acid residues interacting with the ligands are shown as sticks; and magnesium ions are shown as a blue Van der Waals sphere. The square represents the approximate grid box used to perform the docking studies. The analysis of the interactions was carried out using the Protein-Ligand interaction profiler (PLIP)[41] and Discovery Studio. Images were prepared using Pymol 2.4.0. 